\title{
Eyelid Surgery in Facial Palsy
}

\author{
B. LEATHERBARROW ${ }^{1}$ and J. R. O. COLLIN ${ }^{2}$ \\ Manchester and London
}

\begin{abstract}
Summary
Paralysis of the orbicularis oculi muscle is by far the most serious consequence of loss of function of the facial nerve. The severity of the resultant ocular problems is related to the degree and duration of this paralysis. The primary goal of the ophthalmologist in managing the patient with orbicularis oculi paralysis is to protect the cornea. The alleviation of epiphora, correction of paralytic ectropion, and amelioration of the resultant cosmetic deformities are secondary goals.

The major factor in determining the most appropriate therapeutic approach in the management of the patient with a facial paralysis is whether the paralysis is temporary or permanent. In temporary paralysis, conservative methods of treatment are usually adequate and are well documented. In permanent orbicularis paralysis, the development of lid abnormalities is inevitable, and corrective lid procedures are required. The procedures required depend on the resultant dysfunction and degree of deformity.

This study reviews 65 patients who underwent surgical treatment for the ophthalmic consequences of established and permanent facial palsy in order to evaluate the efficacy of their treatment.
\end{abstract}

\section{Patients and methods}

The hospital case notes of 65 patients who had undergone surgical treatment, under the care of the Oculoplastic Unit at Moorfields Eye Hospital, for established and permanent facial nerve palsy were reviewed. The patients had been treated over the period October 1981 to June 1989. The mean age of the patients was 63 years (range $26-88$ years). The age categories of the patients are shown in Table I. There were $29(45 \%)$ males and 36 $(55 \%)$ females. Sixty patients $(92 \%)$ had a unilateral palsy and the remaining five patients $(8 \%)$ had a bilateral palsy. The aetiologies of the facial palsies are shown in Table II. Sixty-three patients $(97 \%)$ had a complete facial paralysis affecting all the facial muscles with a resultant permanent flaccid paralysis. The remaining two patients $(3 \%)$ had a paralysis of the frontalis muscle only with a resultant brow ptosis.

The duration of the palsy prior to surgical treatment under the care of the Oculoplastic Unit was greater than one year in 61 patients $(94 \%)$, and greater than 10 years in 39 patients $(60 \%)$. The mean follow-up period after the final surgical procedure was 12 months (range three months to five years). Only 24 patients $(37 \%)$, however, were followed-up for 12 months or more. Thirty-two patients $(49 \%)$ were followed for more than six months.

Twenty-nine patients ( $45 \%)$ had previously undergone surgical treatment for their facial

From 'Manchester Royal Eye Hospital. ${ }^{2}$ Moorfields Eye Hospital, London.

Correspondence to: Mr. J. R. O. Collin, Consultant Ophthalmic Surgeon, Moorfields Eye Hospital, City Road, London. 
Table I Age categories of patients (years)

\begin{tabular}{ccccc}
\hline $0-20$ & $21-40$ & $41-60$ & $61-80$ & $81-100$ \\
0 & 6 & 19 & 29 & 11 \\
\hline
\end{tabular}

palsy prior to referral to the Oculoplastic Unit. These procedures, which are listed in Table III were performed by ophthalmologists, neurosurgeons, and plastic surgeons.

Four indications for surgical treatment were established: exposure keratopathy, paralytic ectropion, epiphora, and cosmetic deformity. For each individual patient it was noted which of the above indications for treatment were cited, singly or in combination. The types of surgical procedure to which the patients were subjected were recorded. The efficacy of the surgical treatment was judged by evaluating the following parameters, after the surgical treatment was deemed to have been completed:

Objective parameters: Corneal staining Lower lid position

Subjective parameters: Exposure keratopathy symptoms

Epiphora

Cosmesis

Sixty-one patients $(94 \%)$ had an intact Bell's phenomenon. In the remaining four patients $(6 \%)$ this was poor/absent. No patient in the series had a concomitant trigeminal sensory loss with loss of corneal sensation.

\section{Results}

The different surgical procedures to which

Table II Aetiologies of the facial nerve palsies

\begin{tabular}{lc}
\hline Aetiology & No of cases \\
\hline Bell's (idiopathic) palsy & 18 \\
Congenital & 10 \\
Post surgery* & 11 \\
Cerebellopontine angle tumour & 7 \\
Parotid tumour & 4 \\
Moebius syndrome & 2 \\
Post facial nerve avulsion & 2 \\
Ramsay Hunt Syndrome & 1 \\
Mastoiditis & 4 \\
Trauma & 4 \\
Polio & 1 \\
Varicella & 1 \\
\hline
\end{tabular}

*Post surgical treatment of periauricular lesions e.g. carcinoma of external auditory meatus these patients were subjected are listed in Table IV, and descriptions of these can be found in standard ophthalmic and ophthalmic plastic surgical textbooks. ${ }^{2,3}$ The list contains a wide range of soft tissue ophthalmic procedures which have been used on an individual basis after a'careful evaluation of the patients' symptoms and physical signs.

Exposure keratopathy was dealt with in a number of ways. In the presence of upper lid retraction, an under-diagnosed sequel of facial palsy resulting from the unopposed action of the levator palpebrae superioris, an upper lid retractor recession was performed. Where this was greater than $3 \mathrm{~mm}$ a scleral graft 'spacer' was used. Exposure keratopathy affecting principally the inferonasal aspect of the cornea was managed with a Lee medial canthoplasty. ${ }^{4}$ A small permanent lateral tarsorraphy was performed in patients who did not demonstrate significant upper lid retraction.

Paralytic ectropion of the lower lid was managed according to the physical signs observed. The choice of procedure depended on the extent and area of eyelid affected, the presence and extent of medial canthal tendon laxity, and the presence of excess skin or skin shortage. ${ }^{3}$

The management of epiphora mainly involved the reapposition of the lower punctum to the globe. Six patients had a stenosed punctum and were treated with a single snip procedure. Two young patients severely affected by epiphora, despite correction of a lower lid ectropion and reapposition of the lower punctum, were successfully managed with a Jones by-pass tube.

Residual cosmetic deformity following upper and lower lid surgery was mainly due to severe brow ptosis. This was managed with a brow lift procedure. ${ }^{5}$

Table III Previous surgical procedures performed prior to referral

\begin{tabular}{lc}
\hline Procedure & $\begin{array}{c}\text { No of } \\
\text { patients }\end{array}$ \\
\hline Fascia lata sling & 3 \\
Lateral tarsorrhaphy (temporary type) & 16 \\
Upper lid spring & 3 \\
Upper lid gold implant & 3 \\
Regional muscle transfer & 4 \\
\hline
\end{tabular}


Table IV Surgical procedures used in series

\begin{tabular}{lc}
\hline & No \\
Procedures & performed \\
\hline Exposure keratopathy & \\
Lee medial canthoplasty & 42 \\
Upper lid retractor recession & 18 \\
Upper lid recession + scleral graft & 3 \\
Lateral tarsorrhaphy & 17 \\
lower lid ectropion & \\
Lateral tarsal strip/canthal sliing & 16 \\
Medial canthal resection & 12 \\
Medial canthal tendon posterofixation & 4 \\
Lazy-T procedure & 3 \\
Horizontal lower lid shortening & 3 \\
Kuhnt-Symanowski procedure & 2 \\
Lower lid inverting sutures & 1 \\
Skin graft/flap to lower lid & 4 \\
Medial conjunctivoplasty & 5 \\
Epiphora & \\
1 snip to lower punctum & \\
Jones by-pass tube & 6 \\
Cosmesis & 2 \\
Brow lift & \\
Others & 2 \\
Partial opening of tarsorrhaphy & \\
Removal of spring & \\
Removal of gold implant & \\
\hline & \\
\hline
\end{tabular}

The number of separate surgical sessions which the individual patients attended is listed in Table $\mathrm{V}$ and the number of separate surgical procedures to which the individual patients were subjected at single or multiple surgical sessions is listed in Table VI. It can be seen that $49 \%$ of the patients were required to attend more than one operating session and $23 \%$ more than two sessions during the course of their treatment. Seventy-five per cent of the patients were subjected to more than one separate surgical procedure, $57 \%$ more than two procedures.

The postoperative subjective and objective improvements which were achieved are

Table $\mathbf{V}$ The number of separate surgical sessions attended

\begin{tabular}{lc}
\hline No of surgical sessions & No of patients \\
\hline 1 & 33 \\
2 & 17 \\
3 & 12 \\
4 & 2 \\
5 & 0 \\
6 & 1 \\
\hline
\end{tabular}

detailed in Tables VII and VIII respectively. Epiphora remained a significant problem in $42 \%$ of the patients. It can be seen, however, that the surgical treatment was far more successful in improving exposure keratopathy symptoms and signs, paralytic ectropion, and cosmetic deformities.

Of the 29 patients who had undergone the surgical procedures listed in Table III prior to referral, two patients required a re-operation for lateral tarsorrhaphy, one required a partial opening of an extensive tarsorraphy, two had an extruding spring and one an extruding gold implant, all of which were removed.

There were very few reported complications of surgery, one patient was noted to have postoperative anaesthesia of the forehead following a brow lift due to the inadvertant division of the supraorbital nerve. One patient developed a postoperative wound infection following a brow lift. This resolved completely on systemic antibiotics. One further patient, who had undergone an upper lid retractor recession, was overcorrected and had a $3 \mathrm{~mm}$ ptosis. This was later corrected with a posterior approach levator advancement procedure.

\section{Discussion}

It is impossible to provide accurate, complete, and standardised data on the results of surgery for facial palsy from a review of patients' hospital records. Nevertheless, this study, in spite of the limitations inherent in its design, has provided many useful clinical insights.

A review of 65 cases of facial nerve palsy shows that the optimum treatment of the ophthalmic consequences requires multiple procedures over a period of time. Lateral tarsorrhaphy, the standard procedure of the ophthalmic community for corneal protec-

Table VI The number of separate surgical procedurs undergone

\begin{tabular}{lc}
\hline No of procedures & No of patients \\
\hline 1 & 16 \\
2 & 12 \\
3 & 20 \\
4 & 9 \\
5 & 5 \\
6 & 2 \\
7 & 1 \\
\hline
\end{tabular}


Table VII Postoperative subjective improvement

\begin{tabular}{lcccc}
\hline Symptoms & No pre-op & \% Resolved & \% Improved & \% Unchanged \\
\hline Epiphora & 36 & 22 & 36 & 42 \\
Exposure symptoms & 46 & 61 & 24 & 15 \\
Poor cosmesis & 24 & - & 83 & 17 \\
\hline
\end{tabular}

tion, is not a satisfactory procedure when performed alone in the majority of cases. Of the 16 patients who had previously had a lateral tarsorrhaphy, eight underwent a medial canthoplasty, one a medial canthal resection, and the remainder a combined medial canthoplasty and upper lid retractor recession. Preoperatively, the most annoying symptom was epiphora, affecting $55 \%$ of the patients. In none of these patients, however, was epiphora the sole complaint. In facial palsy this is due to a combination of defective tear drainage and increased tear production. An atonic orbicularis oculi muscle cannot supply the dynamic lacrimal pumping mechanism which propels the thin tear meniscus across the lower lid to the canaliculi and lacrimal sac. In addition, exposure keratopathy causes a reflexly increased lacrimal gland output. The complaint of epiphora had been carefully assessed preoperatively to exclude an associated nasolacrimal obstruction. In none of the patients was this found. Following surgery, $42 \%$ were unchanged and only $22 \%$ were symptom-free. Although often regarded as a minor complaint, severe epiphora had resulted in such a degree of lower lid skin excoriation in four patients that a skin graft or flap was required to correct the resultant cicatricial element of their ectropion.

Of the 46 patients who had exposure keratopathy symptoms and signs preoperatively, $15 \%$ were unchanged postoperatively. It is noteworthy that none of the latter underwent an upper lid retractor recession. An upper lid retractor recession was performed on 21 of the 46 patients with exposure keratopathy, all of whom demonstrated upper lid retraction. In three of the patients the degree of retrac- tion was sufficient to require a scleral graft. In 42 of these patients, with exposure keratopathy mainly affecting the medial aspect of the cornea, a Lee type of medial canthoplasty was performed. In 26 of these patients the medial canthoplasty was combined with a lateral tarsorrhaphy/lateral canthal sling/lateral tarsal strip procedure.

A medial canthal resection procedure was substituted for a medial canthoplasty in 12 patients who had a marked laxity of the medial canthal tendon. Table IV shows that a small number of additional lower lid procedures were performed on an individual basis in the absence of exposure keratopathy.

Twenty-four of the patients were primarily concerned with the cosmetic deformity resulting from their facial palsy. Fifteen of these patients had a severe brow ptosis. They underwent a brow elevation procedure as described by Beard. ${ }^{5}$ Only one patient was dissatisfied with the cosmetic improvement postoperatively. Of the remaining nine patients, whose cosmetic deformity was related to their lower lid ectropion, six patients were improved and three were unchanged.

Of the patients who had undergone surgery prior to referral, six had received prosthetic devices to overcome lagophthalmos. Of these, three had to be removed. It has been shown that a very high proportion of eyelid implantation devices fail to work or require reoperation within a relatively short period of time. ${ }^{6,7}$ Those patients who had undergone regional muscle transfers achieved excellent results when combined with lower lid procedures. Lisman et al have shown that the success rate of lower lid ectropion surgery in

Table VIII Postoperative objective improvement

\begin{tabular}{lcccc}
\hline Signs & No pre-op & \% Resolved & \% Improved & \% Unchanged \\
\hline Corneal staining & 46 & 70 & 15 & 15 \\
Lower lid ectropion & 41 & 49 & 36 & 15 \\
\hline
\end{tabular}




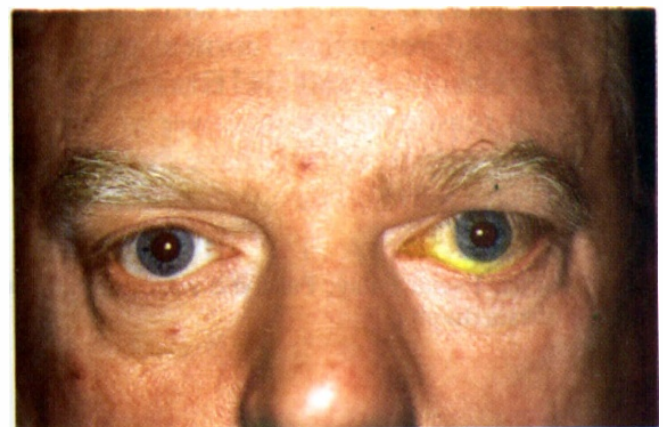

Fig. 1a. Preoperative appearance: Left facial palsy showing upper and lower lid retraction with pooling of fluorescein.

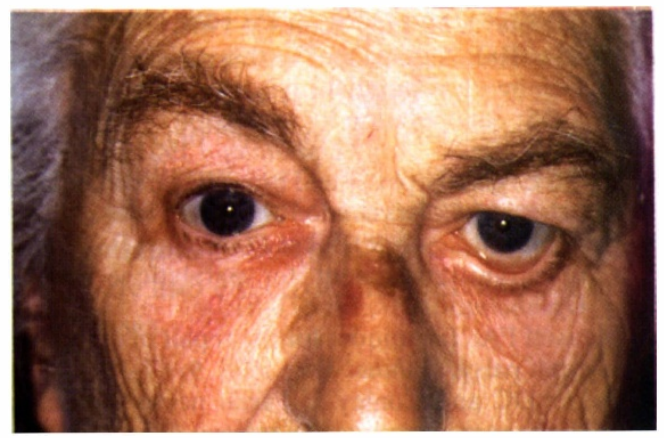

Fig. 2a. Preoperative appearance: Left facial palsy showing a paralytic ectropion and mild brow ptosis.

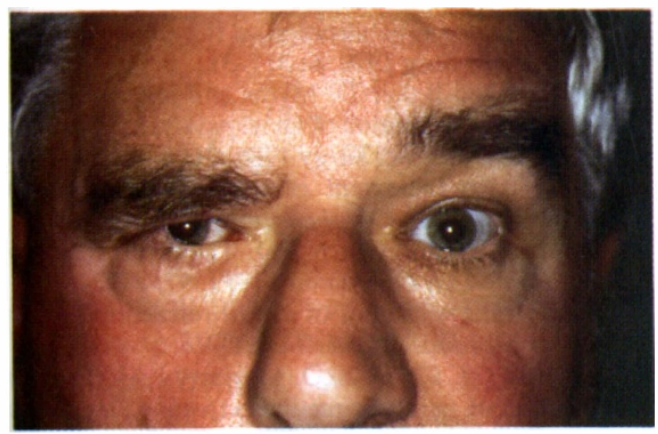

Fig. 3a. Preoperative appearance: Right facial palsy showing a marked brow ptosis and lateral tarsorrhaphy restricting the temporal visual field.

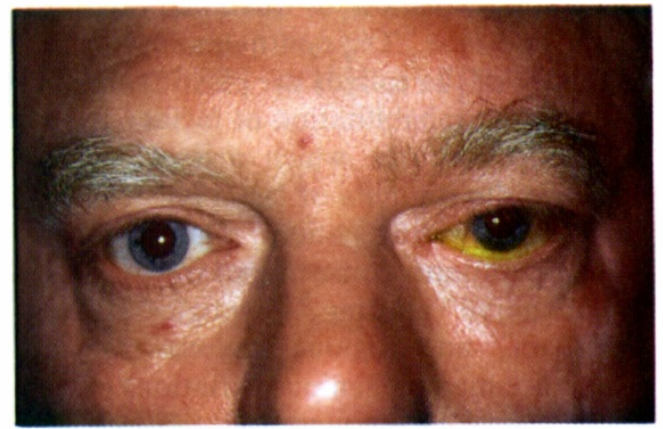

Fig. 1b. Postoperative appearance following a left upper lid retractor recession and medial canthoplasty. The pooling of fluorescein is unchanged.

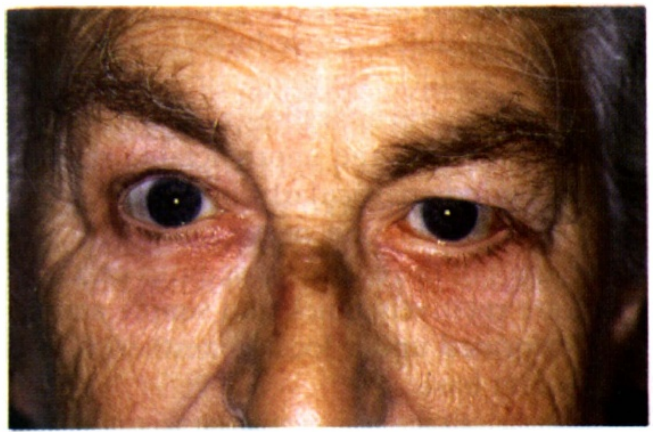

Fig. 2b. Postoperative appearance following a left lower lid lateral tarsal strip procedure.

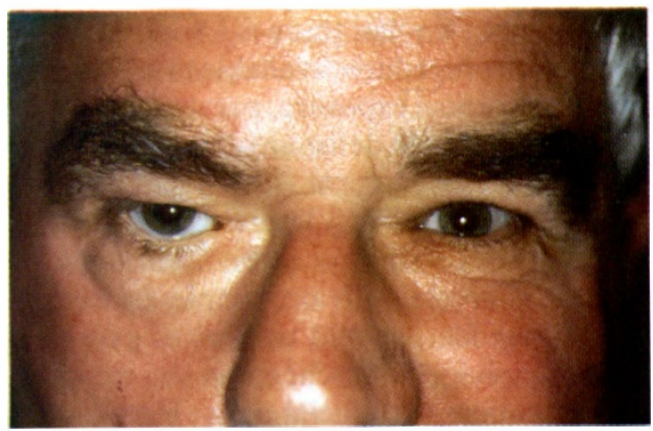

Fig. 3b. Postoperative appearance following a brow lift, opening of tarsorrhaphy, lateral tarsal strip procedure and medial canthoplasty. 
facial palsy is greater if performed in conjunction with elevation or reanimation of the ptotic mid-face. ${ }^{6}$ The ophthalmologist should be aware of the role of the plastic surgeon in the surgical rehabilitation of facial palsy and of the procedures which are available to improve the stigmata of facial palsy, particularly in the young patient. There are three basic operations, consisting of free ipsilateral facial nerve grafting, ${ }^{8}$ hypoglossal nerve crossover, ${ }^{9}$ and regional muscle transfer. ${ }^{10}$ There are many ingenious variations of these fundamental techniques, namely, cross-face anastomosis, crossing over the spinal accessory, phrenic, and descending cervicalis nerves, and free muscle transplants with and without neurovascular microsurgical techniques. ${ }^{11}$

Although these procedures can produce excellent results, they are time consuming requiring general anaesthesia, hospitalisation and often a long and unpredictable rehabilitation period, particularly in those patients who have undergone nerve grafts. By contrast, most of the eyelid procedures described here are relatively quick and simple and can be performed under local anaesthesia on an outpatient basis.

It is evident that the ocular consequences of paralysis of the orbicularis oculi muscle follow a progressive course. It is necessary, therefore, to individualise procedures depending on the degree of deformity and dysfunction. We have shown that there is a wide variety of soft tissue procedures available to successfully manage corneal exposure, paralytic ectropion and the cosmetic deformity. Epiphora is less amenable to surgical improvement. The medial canthoplasty, ${ }^{9}$ lateral canthal sling, ${ }^{12}$ lateral tarsal strip procedure ${ }^{13}$ and upper lid retractor recession ${ }^{14}$ are simple procedures which, we feel, should be part of every surgeon's management scheme in the treatment of the patient with facial palsy. If the medial canthal tendon is very lax, a medial canthal resection reapposes the lower lid effectively. ${ }^{15}$ Many prosthetic devices are available to improve lid closure, ${ }^{16,17,18}$ but their use is limited due to a high complication rate. ${ }^{6}$ The more major general plastic surgery procedures are valuable in maintaining muscle tone and in rehabilitating the whole face, but the minor primarily ophthalmic procedures discussed in this paper are often sufficient alone to correct lid retraction, corneal exposure, paralytic ectropion and the cosmetic deformity with reasonable results over an acceptable follow-up period as we have shown in this paper (Figs. 1-3):

KeY WoRds: Facial Palsy; Paralytic ectropion; Brow ptosis; Tarsorrhaphy.

\section{References}

${ }^{1}$ Jelks GW, Smith B, Bosniak S: The evaluation and management of the eye in facial palsy. Clin Plast Surg 1979, 6: 397-419.

${ }^{2}$ McCord CD: Surgery of the eyelids. In Duane TD ed. Clinical ophthalmology Vol 5 Chap 5. Philadelphia: J. B. Lippincott Co 1988.

${ }^{3}$ Colllin JRO: A manual of systematic eyelid surgery. Edinburgh: Churchill Livingstone 1989.

4 Lee OS: An operation for correction of everted lacrimal puncta. Am J Ophthalmol 1951; 34: 575-8.

${ }^{5}$ Beard C: Canthoplasty and brow elevation for facial palsy. Arch Ophthalmol 1964, 71: 386-8.

${ }^{6}$ Lisman RD, Smith B, Baker D, Arthurs B: Efficacy of surgical treatment for paralytic ectropion. Ophthalmol 1987, 94: 671-81.

${ }^{7}$ Mausolf FA: Techniques for the repair of orbicularis oculi palsy. Ophthal Surg 1978, 9: 67-70.

${ }^{8}$ Baker DC and Conley J: Facial nerve grafting: a thirty-year retrospective review. Clin Plast Surg 1979, 6: 343-000.

${ }^{9}$ Conley J and Baker DC: Hypoglossal-facial nerve anastomosis for reinnervation of the paralysed face. Plast Reconstruct Surg 1979, 63: 63-72.

${ }^{10}$ Baker DC and Conley J: Regional muscle transposition for rehabilitation of the paralysed face. Clin Plast Surg 1979, 6: 317-31.

${ }^{11}$ Conley J and Baker D: Myths and misconceptions on the rehabilitation of facial paralysis (editorial). Plast Reconstruct Surgy 1986, 71: 538-9.

${ }^{12}$ Tenzel RR: Treatment of lagophthalmos of the lower lid. Arch Ophthalmol 1969, 81: 366-8.

${ }^{13}$ Anderson RL and Gordy DD: The tarsal strip procedure. Arch Ophthalmol 1979, 97: 2192-6.

${ }^{14}$ Henderson JW: Relief of eyelid retraction. A surgical procedure. 1965, 74: 205-16.

${ }^{15}$ Crawford GJ, Collin JRO, Moriarty PAJ: The correction of paralytic medial ectropion. $\mathrm{Br} \mathrm{J} \mathrm{Oph}$ thalmol 1984, 68: 639-41.

${ }^{16}$ Smellie GD: Restoration of the blinking reflex in facial palsy by a simple lid-load operation. $B r J$ Plast Surg 1966, 19: 279-83.

${ }^{17}$ Arion HG: Dynamic closure of the lids in paralysis of the orbicularis muscle. Int Surg 1972, 57: $48-50$.

${ }^{18}$ Morel-Fatio D and Lalardrie J-P: Palliative surgical treatment of facial paralysis. The palpebral spring. Plast Reconstruct Surg 1964; 33: 446-56. 